\title{
Long-Term Destiny of Corneal Endothelial Cells in Anterior Chamber Intraocular Lens-Implanted Eyes
}

\author{
Yating Tang $\mathbb{D},^{1,2}$ Jie Xu $\mathbb{D}^{1,2}$ Jiahui Chen $\mathbb{D}^{1,2}$ and Yi Lu $\mathbb{D}^{1,2}$ \\ ${ }^{1}$ Department of Ophthalmology and Eye Research Institute, Eye and ENT Hospital of Fudan University, 83 Fenyang Road, \\ Xuhui District, Shanghai 200031, China \\ ${ }^{2}$ NHC Key Laboratory of Myopia (Fudan University), Key Laboratory of Myopia, \\ Chinese Academy of Medical Science and Shanghai Key Laboratory of Visual Impairment and Restoration, \\ Shanghai 200031, China
}

Correspondence should be addressed to Yi Lu; luyieent@126.com

Received 8 May 2020; Revised 9 December 2020; Accepted 17 December 2020; Published 24 December 2020

Academic Editor: Enrique Mencía-Gutiérrez

Copyright (c) 2020 Yating Tang et al. This is an open access article distributed under the Creative Commons Attribution License, which permits unrestricted use, distribution, and reproduction in any medium, provided the original work is properly cited.

Purpose. To investigate the long-term changes of corneal endothelial cells (EC) in anterior chamber intraocular lens- (AC-IOL-) implanted eyes. Methods. Retrospective study. We included 37 eyes (25 patients) that received AC-IOL implantation previously in the Eye and ENT Hospital of Fudan University between 1995 and 2016. Follow-up outcomes included the best-corrected visual acuity (BCVA), endothelial cell density, hexagonality, coefficient of variance, and central corneal thickness. Results. In total, 23 eyes (62.16\%) with phakic and 14 eyes (37.84\%) with aphakic AC-IOLs were included. Among these, 3 eyes (8.11\%) were anglesupported AC-IOLs and 34 eyes (91.89\%) were Artisan iris-fixated AC-IOLs. The mean age of patients was $41.40 \pm 17.17$ years, and the mean follow-up time was $12.12 \pm 4.71$ years in our study. At the follow-up time, corneal decompensation existed in 3 anglesupported AC-IOL eyes with a rate of $100 \%$ and 15 iris-fixated AC-IOL eyes with a rate of $44.12 \%$. AC-IOL displacement occurred in 14 (41.18\%) iris-fixated AC-IOL eyes. In the 19 iris-fixated AC-IOL eyes without corneal decompensation, significant changes also took place in corneal endothelial cells. The endothelial cell density decreased from $2843.26 \pm 300.76$ to $2015.58 \pm 567.99$ cells/ $\mathrm{mm}^{2}(29.1 \%$ loss, $P<0.001)$ and hexagonality decreased from $51.21 \pm 7.83$ to $42.53 \pm 9.17$ (\%) (16.9\% loss, $\left.P<0.001\right)$. The Kaplan-Meier survival curve also demonstrated the accumulated expectation rates of corneal endothelial cell decomposition for AC-IOLs with a median survival time of 12 years. Conclusion. We reported a significant chronic loss and long-term decompensation destiny of corneal endothelial cells in AC-IOL eyes. Semiannual or annual follow-up and evaluation of endothelial cells should be conducted in AC-IOL-implanted patients.

\section{Introduction}

Anterior chamber intraocular lenses (AC-IOLs) have been widely used in the past two decades in phakic myopic eyes and in aphakic eyes after a cataract extraction procedure. Given the relatively high efficiency and simplicity of the operation, Nuvita angle-supported and Artisan iris-fixated IOLs were the two most commonly and successfully used IOLs across all AC-IOLs in China. Though their effectiveness in correcting refractive errors has been soundly proven [1-3], their long-term safety and reliability are still an unresolved question, as most follow-up data of eyes implanted with angle- supported and iris-fixated IOLs were for less than five years [4-6].

The endothelial cell density is a key criterion for accessing the safety of AC-IOLs. The importance of endothelial cell density (ECD) loss for measuring the safety of the anterior chamber was emphasized by the American Academy of Ophthalmology (AAO) in early 2017 [7]. The AAO Task Force guidelines recommend that the percentage of eyes with a total ECD loss $\geq 25 \%$ after 3 years be used as an endpoint of investigation criterion. However, the AAO did not provide the long-term destiny prediction of AC-IOLs, considering that the data on long-term changes and safety (more than 10 years) of corneal 
endothelial cells were still urgently limited [3, 5, 8, 9]. Hence, in this study, we conducted a retrospective nonrandomized study of angle-supported and iris-fixated IOLs with a mean follow-up time of over 12 years. Our study aimed to provide some predictive data to foresee the long-term trend of corneal endothelial cells in ACIOL-implanted eyes.

1.1. Patients and Methods. Patients who were implanted with angle-supported and iris-fixated AC-IOLs at the Eye and ENT Hospital of Fudan University, Shanghai, between 1995 and 2016 were identified through surgical records $(n=42,57$ eyes). From these patients, 13 could not be reached by telephone and 4 refused to be involved in the study; therefore, from the original group, 25 patients (37 eyes) had follow-up data available. This study adhered to the tenets of the Declaration of Helsinki. Approval was obtained from the Human Ethics Committee of the Eye and ENT Hospital of Fudan University, and all patients signed a statement of informed consent before all treatment and follow-up.

Inclusion criteria were patients older than 20 years at the implantation of Nuvita angle-supported AC-IOLs (B\&L Nuvita MA20, Baush \& Lomb, USA) and older than 6 years for Artisan iris-fixated AC-IOLs (Ophtec B. V., the Netherlands). The Nuvita angle-supported AC-IOL is a onepiece, polymethyl methacrylate (PMMA), rigid lens with a biconcave optical zone of $6 \mathrm{~mm}$. The iris-fixated AC-IOL is a one-piece, PMMA, rigid lens with a convex-concave optic and an optical zone of $5 \mathrm{~mm}$.

All the enrolled patients were angle-supported or irisclaw AC-IOL implanted, had an anterior depth (ACD) at the period of implantation of $3 \mathrm{~mm}$ or more, and had an endothelial cell density $\geq 2000$ cells $/ \mathrm{mm}^{2}$ in patients $\geq 25$ years and $\geq 2500$ cells $/ \mathrm{mm}^{2}$ for younger patients. Exclusion criteria were previous intraocular surgery, glaucoma, eye trauma, uveitis, and corneal diseases that might have had influence on the endothelial cells before the AC-IOL implantation surgery. For aphakic eyes, patients underwent cataract extraction surgery, in addition to the inclusion and exclusion criteria above.

For each patient, we recorded the age at implantation, the age at explantation (if it continued), and age at corneal decompensation (if it occurred). We also recorded patient complications during the whole long-term follow-up (glaucoma, corneal decompensation, uveitis, cataract, and retinal detachment). Best-corrected visual acuity (BCVA), slit-lamp microscopy, intraocular pressure (IOP), and fundus examination after pupil dilation were performed at all follow-up visits. The corneal endothelial cells were measured using the Tomey specular microscope (EM-3000, Tomey, Tokyo, Japan).

The method of implantation of angle-supported and irisfixated AC-IOLs was conducted under local peribulbar anesthesia. All eyes achieved miosis status preoperatively using pilocarpine. All eyes were treated with an identical surgical procedure for the two AC-IOLs. After the $5 \mathrm{~mm}$ corneoscleral incision was centered at 12 o'clock, the viscoelastic substance (Discovisc, Alcon, USA) was injected sufficiently into the anterior chamber to support the anterior chamber depth, to protect the endothelial cells, and to avoid lens-touching during implantation. The angle-supported and iris-fixated IOLs were implanted into the anterior chamber. For angle-supported IOLs, the IOL was inserted in the anterior chamber with the distal haptics directed to the angle. The IOL was gently rotated at the horizontal axis. A slight adjustment of haptics was conducted to avoid pupil ovalization. For Artisan IOLs, after being rotated at the horizontal axis, the claws were fixed to the midperipheral iris stroma. For all eyes, a slit iridotomy was performed at 12 o'clock to avoid pupillary block glaucoma. The viscoelastic substance was exchanged for balanced salt solution, and the incision was sutured with 3-5 interrupted 10-0 nylon sutures. After surgery, topical levofloxacin and prednisolone acetate were used 3 times daily for 3 to 4 weeks.

We analyzed the data using SPSS statistics version 24.0 (IBM/SPSS, Inc., Chicago, IL). Frequency (\%) was used for the analysis of qualitative variables, and the mean and standard deviation (mean \pm SD) were used for the analysis of quantitative variables. The comparisons between preoperative and last follow-up points were performed by paired $t$ test. Kaplan-Meier survival curves were used to compare corneal decompensation rates. Stepwise multivariate logistic regression procedures were conducted to evaluate the independent risk factors of corneal endothelium decompensation. The ORs and 95\% confidence intervals are presented. Statistical significance was defined as $P \leq 0.05$.

\section{Results}

At the last follow up, 25 patients (37 eyes) successfully completed the study. The characteristics of the study population are summarized in Table 1 . The mean follow-up time was $12.12 \pm 4.71$ years. A total of 3 eyes $(8.11 \%)$ were implanted with angle-supported IOLs, and 34 eyes $(91.89 \%)$ were implanted with Artisan IOLs. At the follow-up time, corneal endothelial decompensation occurred in 18 eyes (48.65\%) (Figure 1), with the mean corneal decompensation time of $10.17 \pm 5.19$ years. Corneal decompensation occurred in all 3 angle-supported eyes (100\%) and 15 iris-fixated eyes (44.12\%). AC-IOL displacement occurred in $14(41.18 \%)$ iris-fixated AC-IOL eyes (Figure 1). In the 18 corneal endothelial decompensation eyes, all eyes (100\%) received IOL explantation and 11 eyes (61.11\%) received penetrating keratoplasty (PKP) or Descemet's stripping automated endothelial keratoplasty (DSEK) surgery. Among the 19 irisfixated AC-IOL eyes without corneal decompensation, 16 eyes $(84.21 \%)$ received IOL explantation surgery. In total, 34 eyes (91.89\%) received AC-IOL explantation surgery because of cataracts, marked endothelial cell loss, or corneal decompensation. The mean AC-IOL explantation time was $9.37 \pm 4.45$ years.

For chronic endothelial cell changes in the 19 iris-fixated AC-IOL eyes without corneal decompensation, we also found a significant decrease of ECD and hexagonality (Figure 2(a) and 2(b)). The endothelial cell density decreased from $2843.26 \pm 300.76$ to $2015.58 \pm 567.99$ cells $/ \mathrm{mm}^{2}(29.1 \%$ loss, $P<0.001)$ and hexagonality decreased from $51.21 \pm 7.83$ 
TABLE 1: Demographic data for the enrolled patients.

\begin{tabular}{lcc}
\hline Characteristics & $\begin{array}{c}\text { Angle-supported AC- } \\
\text { IOL }\end{array}$ & Artisan IOL \\
\hline Eye (participates) & $3(3)$ & $34(22)$ \\
Gender (male, \%) & $1(33.33)$ & $13(59.1)$ \\
Age (year) & $41.00 \pm 7.55$ & $41.45 \pm 18.20$ \\
Mean \pm SD (range) & $(34-49)$ & $(13-67)$ \\
Follow-up (year) & $17.33 \pm 5.51$ & $11.41 \pm 4.25$ \\
Mean \pm SD (range) & $(12-23)$ & $(2-20)$ \\
Right eye (\%) & $1(33.33)$ & $20(58.82)$ \\
Phakic AC-IOL (\%) & $3(100)$ & $20(58.82)$ \\
Aphakic AC-IOL (\%) & $0(0)$ & $14(41.18)$ \\
Preop. AXL (mm) & $27.13 \pm 2.24$ & $28.92 \pm 3.99$ \\
Preop. ACD (mm) & $3.52 \pm 0.41$ & $3.54 \pm 0.37$ \\
\hline
\end{tabular}

AXL: axial length; ACD: anterior chamber depth.

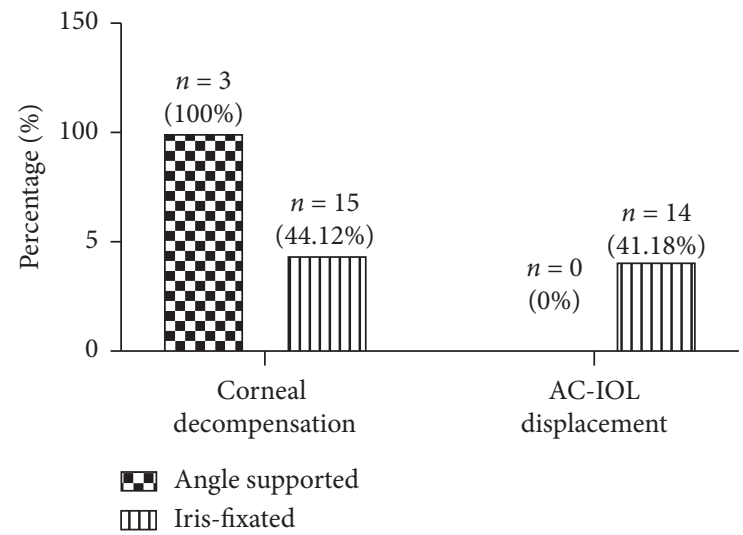

FIgURE 1: Long-term proportion of corneal endothelial decompensation and AC-IOL displacement in angle-supported and irisfixated AC-IOLs. AC-IOLs: anterior chamber intraocular lenses.

to $42.53 \pm 9.17$ (\%) $(16.9 \%$ loss, $P<0.001)$. We also found an increasing trend in the coefficient of variation and central corneal thickness; however, it was not significant (Figures 2(c) and 2(d)).

We next used Kaplan-Meier survival curves to predict the corneal endothelial cell survival rates in AC-IOLimplanted eyes (Figure 3). The first onset of corneal decompensation was 3 years at follow-up, and the median survival time was 12 years. We next used a stepwise multivariate logistic regression model to study the independent risk factors of corneal endothelium decompensation. We included sex, age, preoperative ACD, the duration of time the AC-IOL was in the eye, IOL displacement, glaucoma, and uveitis. However, no significant independent risk factor was found in our model (all $P>0.05$ ).

\section{Discussion}

Reporting the long-term changes of corneal endothelial cells on AC-IOLs is critical to provide evidence for establishing implantation guidelines and follow-up strategies. However, few studies have followed up patients for 10 years or more, and data for Chinese patients are even more rare. In the current study, we conducted a retrospective study of corneal endothelial cell changes with the mean follow-up time of $12.12 \pm 4.71$ years. We found that, at the follow-up time of 12 years, corneal endothelial cell decompensation had occurred in approximately half of the eyes. Furthermore, we also found a significant decrease in ECD (approximately, 30\% loss) and hexagonality (16.9\% loss) in eyes without corneal decompensation. We believe our patient data are valuable for understanding long-term outcomes and the safety of ACIOLs in clinics.

Over the past years, most studies have focused on refractive and visual outcomes of AC-IOLs in myopic and pseudophakic eyes $[10,11]$. In January 2017, the AAO Task Force published a special report with regard to the endothelial cell data for new phakic as well as pseudophakic IOLs. The guideline indicated that eyes with $\geq 25 \%$ ECD loss 3 years after implantation should be considered an endpoint for clinical investigation of Artisan IOL [7]. Another guideline made by the French Health Products and Safety Agency (AFSSAPS) suggested an ECD loss $<1500$ cells $/ \mathrm{mm}^{2}$ or a total EC loss of $30 \%$ as a threshold for AC-IOL explantation [12, 13]. This group assumed that 1500 cells $/ \mathrm{mm}^{2}$ is an important threshold to safely perform phakic IOL explantation and combined cataract surgery without compromising the long-term integrity of the corneal endothelium. However, both guidelines were based on short follow-up times, and most studies on ECD loss were short-term research $[5,14]$. Given that most patients with implanted AC-IOLs were young people, and the 10 years or more of data on the corneal endothelium should also be considered in decision-making for the explantation of AC-IOLs. Jonker et al. [12] indicated that, for iris-fixated IOLs, the median end-point survival time (defined by AAO or AFSSAPS) was 15 years in implanted myopic eyes. Alio et al. [8] found that, for angle-supported IOLs, the median time to explantation was 12.3 years. In our study, we also found that the median survival time of the corneal endothelium was 12 years, which is mainly consistent with previous studies and much earlier than that of Jonker's study. In our study, AC-IOLs were explanted in 34 eyes (91.89\%), with the mean explantation time of $9.37 \pm 4.45$ years. Though the explantation rate was much higher, there were still 18 eyes in which corneal endothelial decompensation occurred and another 2 eyes with ECD $<1500$ cells $/ \mathrm{mm}^{2}$ in the noncorneal decompensation group. We also noticed that the earlier the AC-IOLs were explanted, the less ECD loss happened. Considering the annual linear decrease of ECD in AC-IOLs $[12,15,16]$, we strongly recommend annual or semiannual examination using a specular microscope, and earlier explantation of AC-IOLs of less than 10 years would be helpful.

Several studies have found that a small ACD and a small edge distance were potential risk factors for ECD loss $[15,17,18]$. Jonker [12] and Shajari et al.[18] found that $\mathrm{ACD}<3.00 \mathrm{~mm}$ and small peripheral edge distance $<1.0 \mathrm{~mm}$ were associated with significantly higher ECD loss in short(4 and 5 years) and long-term (10 years) follow-up (HR 5.69 and HR 5.35, respectively, both $P<0.001)$. However, in our study, we did not found that small ACD was an independent 


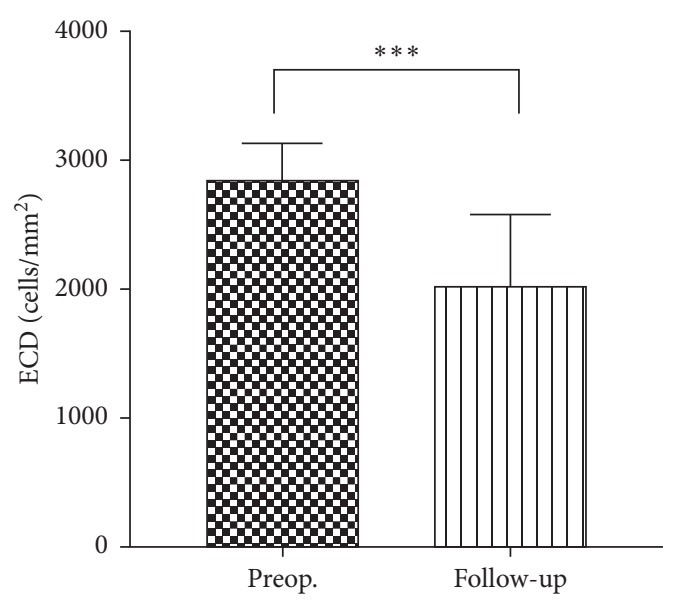

(a)

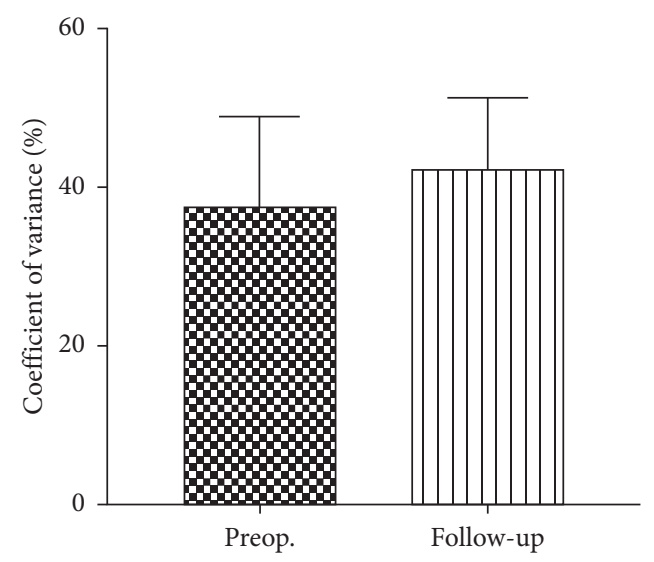

(c)

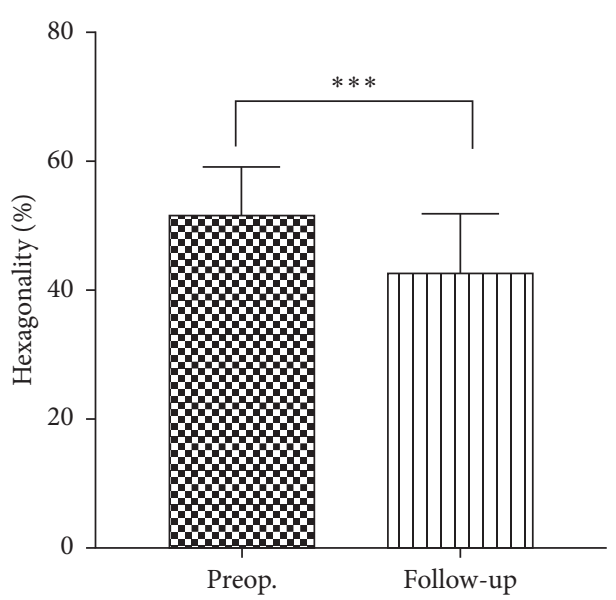

(b)

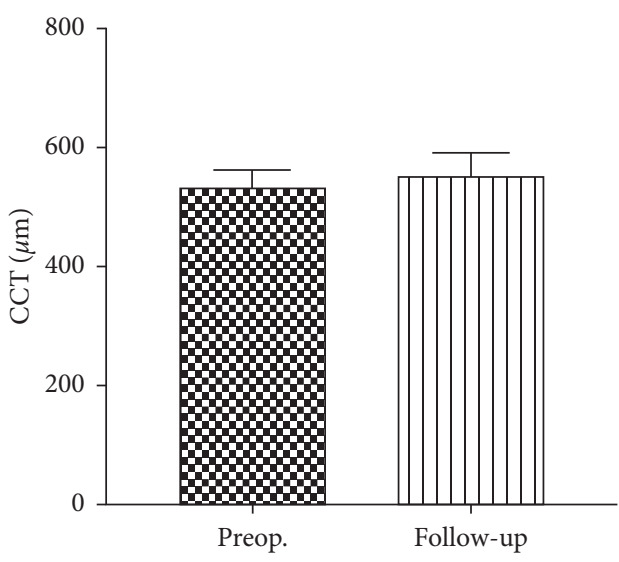

(d)

FIGURE 2: Long-term of ECD, hexagonality, coefficient of variation, and CCT changes in eyes without corneal endothelial cell decompensation. ECD: endothelial cell density; CCT: central corneal thickness.

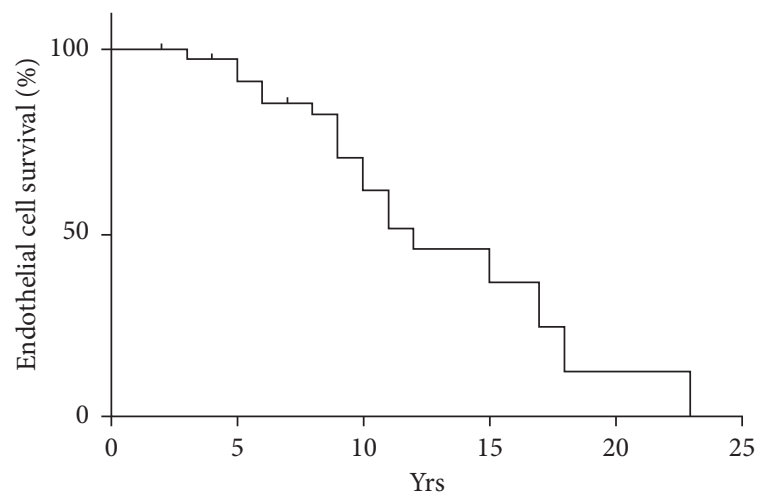

Figure 3: Kaplan-Meier survival curve showing the accumulated expectation rates of corneal decomposition for AC-IOLs. yrs: years.

risk factor for corneal decompensation. Two reasons might contribute to our result. First, we just included ACD $\geq 3 \mathrm{~mm}$ patients when implanting AC-IOLs in our clinical practice and excluded eye trauma and glaucoma patients. Second, most people in our study were much younger (mean age $41.40 \pm 17.17$ years old), and this age difference will result in deeper ACD to some extent. However, we believe that small ACD and edge distance will surely induce higher ECD loss and corneal decompensation because it accelerates the contact and inflammation between the AC-IOL and corneal endothelium, leading to ECD loss and corneal endothelium damage in the end.

Hexagonality proportion is another important indication in our study. Zheng et al. [19] have studied the hexagonal cells in the central region of the cornea by confocal microscopy and found the mean percentage to be $50 \pm 11 \%$, which is consistent with our preoperative data. It has been demonstrated that when endothelial cells are exposed to damage, the hexagonal endothelial cells lose their hexagonal shape and become irregular in shape and size, resulting in the inability to tolerate damage [20]. In our study, we also found a significant decrease in the hexagonal cell proportion over time. In the 18 eyes with corneal endothelium decompensation, the hexagonal cells were 0 ; in eyes without corneal endothelium decompensation, compared with the preoperative data, the hexagonal proportion decreased by $16.9 \%$. We also noticed that, in the corneal endothelium decompensation group, the ECD could be relatively normal (even $>2000$ cells $/ \mathrm{mm}^{2}$ ) but with irregular shape and 0 
hexagonal cells. Thus, we speculated that hexagonality proportion decrease might take place much earlier than ECD loss in a stressed environment. Hexagonality cell decrease might be more objective and accurate for early prediction of ECD loss and corneal endothelium damage. Hexagonal cell change should also be noted in the guidelines and taken into consideration when we make explantation decisions.

The strengths of our study are that it included a relatively large and long-term follow-up with standard examination and detailed data for the eyes of Chinese patients. However, several limitations also exist. This is a retrospective study, and we did not measure the preoperative refraction. Furthermore, considering the fact that some patients did not complete regular annual follow-up, in some cases, we obtained the corneal decompensation onset time from the patients' memory and complaints, and thus, the corneal decompensation diagnosis might have been slightly delayed.

In conclusion, this study demonstrated that, after the implantation of AC-IOLs, the long-term changes of the corneal endothelium were not positive and were somewhat more disappointing than the short-term results. We strongly recommend regular annual or semiannual follow-up to be conducted in patients after AC-IOL implantation. International guidelines are urgently needed to set the threshold regarding ECD and hexagonal cell loss in the case of corneal decompensation in the long run.

\section{Data Availability}

The data used to support the findings of this study are available from the corresponding author upon request.

\section{Disclosure}

This manuscript has been presented at the 24th Congress of Chinese Ophthalmological Society.

\section{Conflicts of Interest}

The authors declare no conflicts of interest.

\section{Acknowledgments}

The work was supported by grants from the Natural Science Foundation of China (NSFC81670835 and NSFC81600719), Shanghai Science and Technology Commission (11231200602), and Shanghai Key Laboratory of Visual Impairment and Restoration (12DZ2260500).

\section{Supplementary Materials}

Original data of the manuscript. (Supplementary Materials)

\section{References}

[1] J. L. Alio, P. Peña-García, K. Pachkoria, J. L. Alio, and A. El Aswad, "Intraocular optical quality of phakic intraocular lenses: comparison of angle-supported, iris-fixated, and posterior chamber lenses," American Journal of Ophthalmology, vol. 156, no. 4, pp. 789-799, 2013.
[2] D. E. Hirashima, E. S. Soriano, R. L. Meirelles, G. N. Alberti, and W. Nosé, "Outcomes of iris-claw anterior chamber versus iris-fixated foldable intraocular lens in subluxated lens secondary to marfan syndrome," Ophthalmology, vol. 117, no. 8, pp. 1479-1485, 2010.

[3] N. G. Tahzib, R. M. Nuijts, W. Y. Wu, and C. J. Budo, "Longterm study of artisan phakic intraocular lens implantation for the correction of moderate to high myopia," Ophthalmology, vol. 114, no. 6, pp. 1133-1142, 2007.

[4] S. Benedetti, V. Casamenti, and M. Benedetti, "Long-term endothelial changes in phakic eyes after Artisan intraocular lens implantation to correct myopia," Journal of Cataract \& Refractive Surgery, vol. 33, no. 5, pp. 784-790, 2007.

[5] M. Pop and Y. Payette, "Initial results of endothelial cell counts after artisan lens for phakic eyes," Ophthalmology, vol. 111, no. 2, pp. 309-317, 2004.

[6] L. Akcay, I. Eser, A. T. Kaplan, A. Taskiran-Comez, and O. K. Dogan, "Phakic anterior chamber lenses in very high myopia: an 18-month follow up," Clinical \& Experimental Ophthalmology, vol. 40, no. 3, pp. 275-281, 2012.

[7] S. MacRae, J. T. Holladay, G. Hilmantel et al., "Special report: American Academy of Ophthalmology Task Force recommendations for specular microscopy for phakic intraocular lenses," Ophthalmology, vol. 124, no. 1, pp. 141-142, 2017.

[8] J. L. Alio, A. Abbouda, P. Peña-Garcia, and S. Huseynli, "Follow-up study of more than 15 Years of an anglesupported phakic intraocular lens model (ZB5M) for high myopia," JAMA Ophthalmology, vol. 131, no. 12, pp. 1541-1546, 2013.

[9] T. Kohnen, L. LaFontaine, R. Andrew et al., "Long-term safety follow-up of an anterior chamber angle-supported phakic intraocular lens," Journal of Cataract \& Refractive Surgery, vol. 43, no. 9, pp. 1163-1170, 2017.

[10] D. Z. Reinstein, T. J. Archer, and J. B. Randleman, "JRS standard for reporting astigmatism outcomes of refractive surgery," Journal of Refractive Surgery, vol. 30, no. 10, pp. 654-659, 2014.

[11] C. Budo, J. C. Hessloehl, M. Izak et al., "Multicenter study of the Artisan phakic intraocular lens," Journal of Cataract \& Refractive Surgery, vol. 26, no. 8, pp. 1163-1171, 2000.

[12] S. M. R. Jonker, T. T. J. M. Berendschot, A. E. Ronden, I. E. Y. Saelens, N. J. C. Bauer, and R. M. M. A. Nuijts, "Longterm endothelial cell loss in patients with artisan myopia and artisan toric phakic intraocular lenses," Ophthalmology, vol. 125, no. 4, pp. 486-494, 2018.

[13] P. F. M. Bernard, Definitive stop of marketing, product recall and follow-up of implanted patients. Presbyopic intraocular lenses NEWLIFE/VIVARTE PRESBYOPIC. Agence Française de Sécurité Sanitaire des Produits de Santé (AFSSAPS), 2007.

[14] R. Maloney, L. H. Nguyen, and M. E. John, "Artisan phakic intraocular lens for myopia Short-term results of a prospective, multicenter study," Ophthalmology, vol. 109, no. 9, pp. 1631-1641, 2002.

[15] M. Doors, T. T. J. M. Berendschot, C. A. B. Webers, and R. M. M. A. Nuijts, "Model to predict endothelial cell loss after iris-fixated phakic intraocular lens implantation," Investigative Opthalmology \& Visual Science, vol. 51, no. 2, pp. 811-815, 2010.

[16] N. Allemann, W. Chamon, H. M. Tanaka et al., "Myopic angle-supported intraocular lenses Two-year follow-up," Ophthalmology, vol. 107, no. 8, pp. 1549-1554, 2000.

[17] M. Doors, D. W. J. K. Cals, T. T. J. M. Berendschot et al., "Influence of anterior chamber morphometrics on endothelial 
cell changes after phakic intraocular lens implantation," Journal of Cataract \& Refractive Surgery, vol. 34, no. 12, pp. 2110-2118, 2008.

[18] M. Shajari, M. Scheffel, M. J. Koss, and T. Kohnen, "Dependency of endothelial cell loss on anterior chamber depth within first 4 years after implantation of iris-supported phakic intraocular lenses to treat high myopia," Journal of Cataract \& Refractive Surgery, vol. 42, no. 11, pp. 1562-1569, 2016.

[19] T. Zheng, Q. Le, J. Hong et al., "Comparison of human corneal cell density by age and corneal location: an in vivo confocal microscopy study," BMC Ophthalmol, vol. 16, p. 109, 2016.

[20] D. S. A. D. Foster and C. H. Dohlman, Smolin and Thoft's the Cornea: Scientific Foundations and Clinical Practice, Lippincott Williams \& Wilkins, vol. 2004p. 48, 4th edition, Philadelphia, PA, 2004. 